\title{
First principles study of electronic and mechanical properties of molybdenum selenide type nanowires
}

\author{
D. Çakır, E. Durgun, O. Gülseren, and S. Ciraci* \\ Department of Physics, Bilkent University, Ankara 06800, Turkey \\ and UNAM-National Nanotechnology Research Center, Bilkent University, Ankara 06800, Turkey
}

(Received 4 August 2006; revised manuscript received 21 October 2006; published 20 December 2006)

\begin{abstract}
Using the first-principles plane-wave pseudopotential method within density functional theory, we have systematically investigated structural, electronic, and mechanical properties of $M_{2} Y_{6} X_{6}, Y_{6} X_{6}(X=\mathrm{Se}, \mathrm{Te}, \mathrm{S}$; $Y=\mathrm{Mo}, \mathrm{Cr}, \mathrm{W}$; and $M=\mathrm{Li}, \mathrm{Na}$ ) nanowires and bulk phase of $M_{2} Y_{6} X_{6}$. We found that not only $\mathrm{Mo}_{6} X_{6}$, but also transition metal and chalcogen atoms lying in the same columns of Mo and Se can form stable nanowires consisting of staggered triangles of $Y_{3} X_{3}$. We have shown that all wires have nonmagnetic ground states in their equilibrium geometry. Furthermore, these structures can be either a metal or semiconductor depending on the type of chalcogen element. All $Y_{6} X_{6}$ wires with $X=$ Te atom are semiconductors. Mechanical stability, elastic stiffness constants, breaking point, and breaking force of these wires have been calculated in order to investigate the strength of these wires. Ab initio molecular dynamic simulations performed at $500 \mathrm{~K}$ suggest that overall structure remains unchanged at high temperature. Adsorption of $\mathrm{H}, \mathrm{O}$, and transition metal atoms like $\mathrm{Cr}$ and $\mathrm{Ti}$ on $\mathrm{Mo}_{6} \mathrm{Se}_{6}$ have been investigated for possible functionalization. All these elements interact with $\mathrm{Mo}_{6} \mathrm{Se}_{6}$ wire forming strong chemisorption bonds, and a permanent magnetic moment is induced upon the adsorption of $\mathrm{Cr}$ or $\mathrm{Ti}$ atoms. Molybdenum selenide-type nanowires can be alternative for carbon nanotubes, since the crystalline ropes consisting of one type of $\left(M_{2}\right) Y_{6} X_{6}$ structures can be decomposed into individual nanowires by using solvents, and an individual nanowire by itself is either a metal or semiconductor and can be functionalized.
\end{abstract}

DOI: $10.1103 /$ PhysRevB.74.235433

PACS number(s): 73.63.Nm, 73.22.-f, 75.75.+a

\section{INTRODUCTION}

In spite of active research carried out on the mechanical, electronic, and transport properties of nanowires for several decades, ${ }^{1-3}$ fabricating interconnects which fit molecular electronic devices have remained one of the major challenges in nanotechnology. Recently, carbon nanotubes ${ }^{4}$ have been a focus of attention because of their diameter in nanometer scale and uniform cylindrical shape allowing ballistic transport. ${ }^{5-7}$ These tubes can be metallic or semiconducting depending on the diameter and chirality angle. They are strong along the axial direction, but flexible radially. Moreover, they can be coated with metal atoms. ${ }^{8-10}$ Nowadays, carbon nanotubes are synthesized in bundles which consist of a large number of tubes of different radius and chiral angle. It seems impossible to fabricate carbon nanotubes in desired diameter and chiral angle or select them from bundles. This difficulty remains a serious obstacle facing the wide range of possible technological applications of carbon nanotubes.

Molybdenum based nanowires with stoichiometry $M_{2} Y_{6} X_{6}$ (where $M=\mathrm{Li}, \mathrm{Na}, Y=\mathrm{Mo}$, and $X=\mathrm{Se}, \mathrm{Te}, \mathrm{S}$ ) have been synthesized by Potel ${ }^{11}$ and Tarascon. ${ }^{12}$ These materials grow also in ropes of structurally and electronically identical nanowires. Each rope has crystalline structure in a hexagonal close packed form. A weak interaction holds the wires together. The isolated wires are easily obtained from the bundles by using a highly polar solvent. ${ }^{13}$ Although these materials are in bulk crystalline form, they show onedimensional (1D) properties due to the existence of long $Y_{6} X_{6}$ atomic chains. Chew et al. ${ }^{14}$ have used the nuclear magnetic-resonance spectroscopy to probe the electronic structure of the $M$ cation sites in $M_{2} \mathrm{Mo}_{6} \mathrm{Se}_{6}, \quad M$ $=(\mathrm{Li}, \mathrm{Rb}, \mathrm{Cs}, \mathrm{In})$. Tarascon et al. ${ }^{12}$ have reported that charge transport properties of $M_{2} \mathrm{Mo}_{6} X_{6}(M=\mathrm{K}, \mathrm{Rb}, \mathrm{Cs}, \mathrm{Tl}$ and $X$ $=\mathrm{Se}, \mathrm{Te}$ ) nanowires can vary from metallic to semiconducting, depending on the temperature and composition. Recently, Venkataraman et al. ${ }^{15,16}$ have synthesized and characterized the structure and electronic properties of the bulk phase of $\mathrm{Li}_{2} \mathrm{Mo}_{6} \mathrm{Se}_{6}$ and isolated $\mathrm{Mo}_{6} \mathrm{Se}_{6}$ chains. Their observations have confirmed the 1D nature of these materials. Local density of states (LDOS) is consistent with Van Hove singularities peculiar to 1D systems. They have also observed that Peierls transition does not occur at least down to $5 \mathrm{~K}$. Venkataraman et al. ${ }^{16}$ have observed Luttinger liquid behavior in $\mathrm{Mo}_{6} \mathrm{Se}_{6}$ nanowires, where linear conductance and current exhibit a power law dependence on temperature and bias voltage, respectively. Exponent governing of these power laws decreases with increasing number of conducting channels. Mechanical and electronic properties of $\mathrm{Li}_{2} \mathrm{Mo}_{6} \mathrm{Se}_{6}$ and individual $\mathrm{Mo}_{6} \mathrm{Se}_{6}$ nanowires have been studied by Riberio et al. ${ }^{17}$ Electronic structure of $\mathrm{Li}_{2} \mathrm{Mo}_{6} \mathrm{Se}_{6}$, isolated $\mathrm{Mo}_{6} \mathrm{Se}_{6}$ wires, and bulk $\mathrm{Li}_{2} \mathrm{Mo}_{6} \mathrm{Se}_{6}$ are similar. Lithium atom behaves as a donor atom. Adsorption of Li does not modify the overall picture of the band structure, since the alkali atom, having very low ionization potential, easily donates its valence electron to the conduction band. Vilfan ${ }^{18,19}$ has studied electronic and elastic properties of bare and $\mathrm{Li}$ doped $\mathrm{Mo}_{6} \mathrm{~S}_{6}$ nanowires with ab initio calculations. $\mathrm{Mo}_{6} \mathrm{~S}_{6}$ wires also weakly interact in bulk form like $\mathrm{Mo}_{6} \mathrm{Se}_{6}$. The elastic constant of these wires along the wire axis is 320 Gpa which is higher than steel but smaller than the single wall carbon nanotubes (SWNT). ${ }^{20,21}$ Recently, $\mathrm{Mo}_{6} \mathrm{~S}_{9-x} \mathrm{I}_{x}$ wires have been synthesized. ${ }^{22}$ The structure of these wires consists of a 
(a)

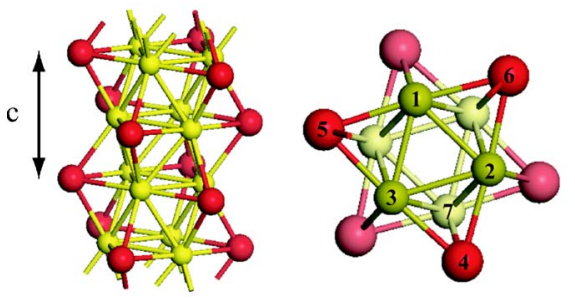

(c)

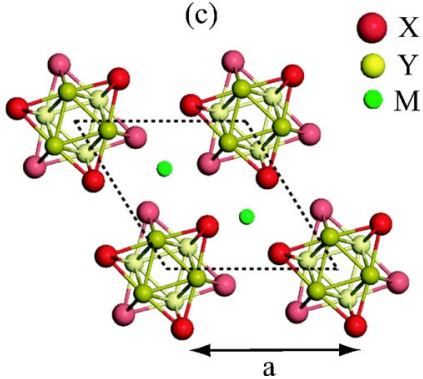

FIG. 1. (Color online) (a) Side and (b) top view of the atomic structure of isolated wires $Y_{6} X_{6} . Y=\mathrm{Mo}, \mathrm{Cr}, \mathrm{W}$; and $X=\mathrm{S}, \mathrm{Se}$, Te. The metal and chalcogen atoms labeled as $(1,2,3)$ and $(4,5,6)$, respectively, are on the same plane. Relevant interatomic distances $d_{1-2}, d_{1-3}, d_{2-3}$, $d_{3-4}, d_{4-7}$, and $d_{7-3}$ are between the atoms (1-2), (1-3), (2-3), (3-4), (4-7), and (7-3), respectively. (c) The unit cell of bulk (periodic) structure of $M_{2} Y_{6} X_{6}$ where $M=\mathrm{Li}$ or Na are in different planes.

rigid Mo octahedra. Each Mo atom is decorated with S or I atoms and octahedras are again connected by three $\mathrm{S}$ or I atoms. Yet, exact structure of these wires is unknown. Yang et $a .^{23}$ have theoretically studied equilibrium geometry and electronic structure of $\mathrm{Mo}_{6} \mathrm{~S}_{9} \mathrm{I}_{9}$ nanowires. Different isomers of these wires exhibit different electrical and magnetic properties. They can become either conductors or narrow gap magnetic semiconductors. Moreover, thin films of metallic $\mathrm{Li}_{2} \mathrm{Mo}_{6} \mathrm{Se}_{6}$ nanowire ropes can be used as a chemical sensor. Xiubin et al. $^{24,25}$ have showed that resistance of such a thin film changes upon adsorption of organic molecules, suggesting that the electrical conductance of the $\mathrm{Mo}_{6} \mathrm{Se}_{6}$ nanowire may change with the type and number of adsorbed atoms or molecules.

Because of their synthesis as structurally identical wires with useful electronic and mechanical properties, $\mathrm{Mo}_{6} \mathrm{Se}_{6}$-type nanowires can be a potential alternative to carbon nanotubes. In this respect, we believe that $Y_{6} X_{6}$-type nanowires will be a subject of interest in the future. This work presents a systematic investigation of structural, mechanical, and electronic properties of $M_{2} Y_{6} X_{6}, Y_{6} X_{6}(X$ $=\mathrm{Se}, \mathrm{Te}, \mathrm{S} ; Y=\mathrm{Mo}, \mathrm{Cr}, \mathrm{W}$; and $M=\mathrm{Li}, \mathrm{Na})$ type nanowires and bulk phase of $M_{2} Y_{6} X_{6}$, within the density functional theory. Our results are important for further research on these nanowires to reveal their properties for practical technological applications.

\section{DESCRIPTION OF THE METHOD}

We have performed first-principles plane-wave calculations $^{26,27}$ within density functional theory (DFT) ${ }^{28}$ using ultrasoft pseudopotentials. ${ }^{29}$ The exchange correlation potential has been approximated by generalized gradient approximation (GGA).$^{30}$ For partial occupancies, we have used the Methfessel-Paxton smearing method. ${ }^{31}$ The width of smearing has been chosen as $0.1 \mathrm{eV}$ for atomic relaxation and $0.01 \mathrm{eV}$ for accurate energy band and electronic density of states calculations. All structures have been treated by supercell geometry (with lattice parameters $a_{s c}, b_{s c}$, and $c_{s c}$ ) using periodic boundary conditions. To prevent interaction between adjacent isolated wires, a large spacing $\left(a_{s c}=b_{s c}\right.$ $\sim 15 \AA$ ) has been taken. For single wire calculations, $c_{s c}$ corresponds to the lattice constant $c_{0}\left(c_{0}\right.$ being the lattice constant of the $Y_{6} X_{6}$ along the wire axis or $z$ direction), but $c_{s c}=2 c_{0}$ for double cell. Convergence with respect to the number of plane waves used in expanding Bloch functions and $\mathbf{k}$ points in sampling the Brillouin zone have been tested for each wire system. In the self-consistent potential and total energy calculations, the Brillouin zone of nanowires has been sampled by $(1 \times 1 \times 15)$ and $(1 \times 1 \times 9)$ mesh points in the $\mathbf{k}$ space within the Monkhorst-Pack scheme ${ }^{32}$ for single and double cells, respectively, since there is no dispersion in $x$ and $y$ directions. For bulk calculations, we have used a grid of $(3 \times 3 \times 15) \mathbf{k}$ points. A plane-wave basis set with kinetic energy cutoff $\hbar^{2}|\mathbf{k}+\mathbf{G}|^{2} / 2 m \leqslant 300 \mathrm{eV}$ has been used. All atomic positions and lattice parameters have been optimized by using the conjugate gradient method where total energy and atomic forces are minimized. The convergence for energy has been chosen as $10^{-5} \mathrm{eV}$ between two ionic steps, and the maximum force allowed on each atom is $0.05 \mathrm{eV} / \AA$.

\section{STRUCTURE}

The overall atomic structure of $\mathrm{Mo}_{6} \mathrm{Se}_{6}$-type nanowires and their bulk crystalline are described in Fig. 1. The unit cell of an isolated nanowire consists of two staggered triangular layers composed of $Y_{3} X_{3}$ units. Each layer consists of an equilateral triangle having one chalcogen atom at each corner and one metal atom placed between each chalcogen pair. Bulk structure unit cell has hexagonal symmetry, where nanowires along the $z$ direction are arranged to form hexagons. Alkali atoms ( $\mathrm{Li}$ or $\mathrm{Na}$ ) are placed between atomic planes as illustrated in Fig. 1(c).

We first study the equilibrium structural properties of isolated nanowires and bulk structures by using total energy calculations where the atomic structure is fully optimized. Our detailed analysis reveals that the geometric structure in Fig. 1 is not unique to the experimentally synthesized $\mathrm{Mo}_{6} \mathrm{Se}_{6}$ compound, but other metal and chalcogen atoms of the same columns can also form such structures. The binding energy $E_{b}$ and the lattice constant $c_{0}$ of optimized structure of $Y_{6} X_{6}$ nanowires are given in Table I. The lattice constants we have calculated for $\mathrm{Mo}_{6} \mathrm{Se}_{6}, \mathrm{Mo}_{6} \mathrm{~S}_{6}$, and bulk $\mathrm{Li}_{2} \mathrm{Mo}_{6} \mathrm{Se}_{6}$ are consistent with those reported in earlier experimental and theoretical works. Experimental value of $c_{0}$ for isolated $\mathrm{Mo}_{6} \mathrm{Se}_{6}$ is $4.5 \pm 0.1 \AA .{ }^{15}$ Vilfan $^{18}$ has calculated lattice con- 
TABLE I. Optimized lattice constant $c_{0}$ (in $\AA$ ), bond lengths $d_{i, j}$ (in $\AA$ ), binding energies $E_{b}$ (in eV/f.u.) of isolated $Y_{6} X_{6}$ nanowires, $E_{c}(X, Y)$ and $E_{b c}$ (binding energy in $\mathrm{eV}$ relative to the bulk cohesive energies). The definitions of $E_{b}, E_{c}(X, Y)$ and $E_{b c}$ are given in the text.

\begin{tabular}{lcccccccccc}
\hline \hline Structure & $c_{o}$ & $d_{1-2}$ & $d_{1-3}$ & $d_{2-3}$ & $d_{3-4}$ & $d_{4-7}$ & $d_{7-3}$ & $E_{b}$ & $E_{c}(X, Y)$ & $E_{b c}$ \\
\hline $\mathrm{Cr}_{6} \mathrm{~S}_{6}$ & 4.03 & 2.51 & 2.52 & 2.54 & 2.33 & 2.36 & 2.49 & 7.90 & 6.27 & 1.63 \\
$\mathrm{Cr}_{6} \mathrm{Se}_{6}$ & 4.14 & 2.43 & 2.46 & 2.5 & 2.46 & 2.50 & 2.52 & 7.11 & 6.32 & 0.79 \\
$\mathrm{Cr}_{6} \mathrm{Te}_{6}$ & 4.23 & 2.41 & 2.42 & 2.43 & 2.68 & 2.70 & 2.54 & 6.40 & 6.12 & 0.29 \\
$\mathrm{Mo}_{6} \mathrm{~S}_{6}$ & 4.35 & 2.75 & 2.77 & 2.76 & 2.48 & 2.52 & 2.69 & 10.52 & 8.93 & 1.59 \\
$\mathrm{Mo}_{6} \mathrm{Se}_{6}$ & 4.44 & 2.72 & 2.75 & 2.74 & 2.61 & 2.64 & 2.72 & 9.82 & 8.99 & 0.83 \\
$\mathrm{Mo}_{6} \mathrm{Te}_{6}$ & 4.58 & 2.66 & 2.66 & 2.66 & 2.81 & 2.86 & 2.76 & 9.18 & 8.79 & 0.40 \\
$\mathrm{~W}_{6} \mathrm{~S}_{6}$ & 4.35 & 2.75 & 2.75 & 2.75 & 2.51 & 2.53 & 2.69 & 12.23 & 11.02 & 1.20 \\
$\mathrm{~W}_{6} \mathrm{Se}_{6}$ & 4.44 & 2.71 & 2.75 & 2.74 & 2.63 & 2.66 & 2.72 & 11.55 & 11.08 & 0.48 \\
$\mathrm{~W}_{6} \mathrm{Te}_{6}$ & 4.55 & 2.67 & 2.67 & 2.67 & 2.85 & 2.87 & 2.75 & 10.89 & 10.87 & 0.01 \\
\hline \hline
\end{tabular}

stant and in-plane Mo-Mo, Mo-S, and interplane Mo-Mo and Mo-S distances of an isolated $\mathrm{Mo}_{6} \mathrm{~S}_{6}$ wire as $4.35 \pm 0.05$, $2.74,2.49,2.69$, and $2.62 \AA$, respectively. These structural parameters are in agreement with our calculated structure parameters for $\mathrm{Mo}_{6} \mathrm{~S}_{6} . E_{b}$ per formula unit (f.u) of isolated wire is calculated as

$$
E_{b}=\left(6 E_{a}[X]+6 E_{a}[Y]-E_{T}\left[Y_{6} X_{6}\right]\right) / 6,
$$

where $E_{a}[X]$ and $E_{a}[Y]$ are the ground state energies of single chalcogen and metal atoms, respectively, and $E_{T}\left[Y_{6} X_{6}\right]$ is the optimized total energy of a nonmagnetic nanowire. High $E_{b}$ obtained for each nanowire can provide some clues about stability of the system. Furthermore, we compare $E_{b}$ of $Y_{6} X_{6}$ wires with $E_{c}(X, Y)$, which is defined as the sum of bulk cohesive energies of each element forming the $Y_{6} X_{6}$ wire. Accordingly, $E_{c}[X, Y]=E_{c}[X]+E_{c}[Y]$, where $E_{c}[X]$ and $E_{c}[Y]$ are the calculated cohesive energy per atom of $X$ and $Y$ bulk crystals, respectively. ${ }^{33,34}$ Binding energies of $Y_{6} X_{6}$ nanowires per f.u. with respect to the cohesive energy of metal and chalcogen crystals are calculated as

$$
E_{b c}=E_{b}-E_{c}(X, Y) .
$$

Calculated $E_{b c}$ and $E_{c}(X, Y)$ are presented in Table I. $E_{b c}$ of $Y_{6} X_{6}$ nanowires ranges from $0.01 \mathrm{eV}$ to $1.63 \mathrm{eV}$, and hence the formation of them from bulk is exothermic. These results indicate that $Y_{6} X_{6}$ nanowires can be produced from bulk precursors.

It is interesting to note that $E_{b}$ increases as the row number of metal atom increases. On the other hand, in each metal group, $E_{b}$ decreases with increasing row number of chalcogen atom since transition metal (TM) atoms are located between two chalcogen atoms occupying the corners of the large triangle, larger radius of metal but smaller radius of chalcogen enhances the binding energy. The variation in $E_{b}$ (and also $E_{b c}$ ) shows the similar trend in the variation of cohesive energies of these elements in bulk phase (see Fig. 2). ${ }^{35}$ For the bulk structure, the optimized lattice parameters are $a=8.52$ and $c_{0}=4.5 \AA$ for $\mathrm{Li}_{2} \mathrm{Mo}_{6} \mathrm{Se}_{6}$. These values are consistent with the experimental values ${ }^{11,16}$ of $a=8.5$ and $c_{0}=4.48 \AA$. Lattice parameter $c_{o}$ of isolated wire changes by $1.4 \%$ in the bulk crystal. The amounts of change in the in- teratomic distances of $\mathrm{Mo}_{6} \mathrm{Se}_{6}$ nanowire are also small upon formation of crystal. It is noted that the variation of the lattice constant of the nanowires follows the same trend of the bulk crystal structure parameters of the corresponding metal atoms (Y). The nature of interaction holding $\mathrm{Li}_{2} \mathrm{Mo}_{6} \mathrm{Se}_{6}$ in bulk phase is conjectured to be attractive van der Waals interaction. In principles, DFT includes the van der Waals interaction. However, it is poorly represented in GGA, but relatively better represented in LDA. In addition to GGA calculations which predicted $c_{o}$ and $a$ of $\mathrm{Li}_{2} \mathrm{Mo}_{6} \mathrm{Se}_{6}$ crystal in good agreement with experimental data, we have also carried out LDA calculations using Ceperley-Alder exchangecorrelation potential. ${ }^{36}$ LDA results underestimate $c_{o}$ and $a$. On the other hand, the smallest Se-Se, Se-Li, and Mo-Se interatomic distances between adjoint wires in bulk phase are

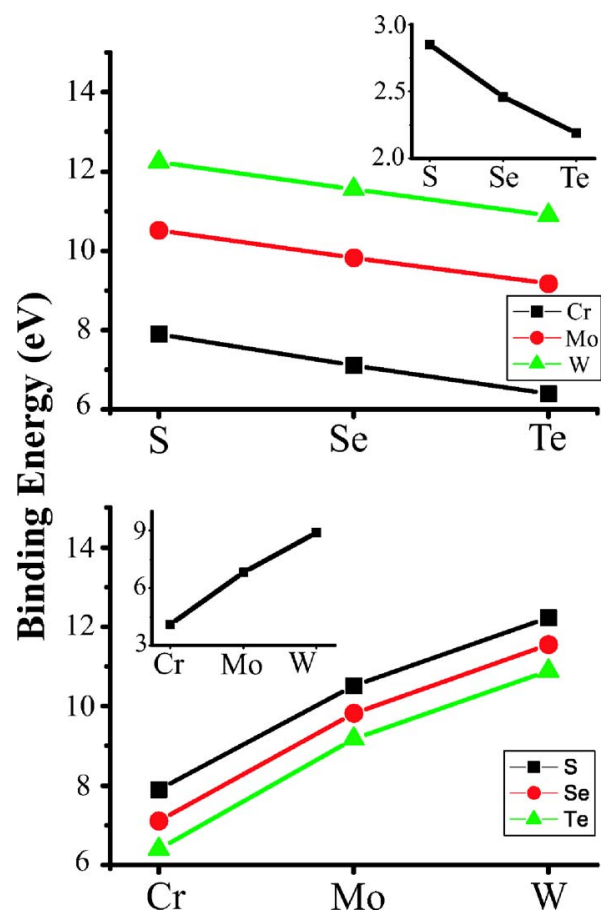

FIG. 2. (Color online) Variation of binding energy $E_{b}$ of $Y_{6} X_{6}$ nanowires with respect to different $X$ and $Y$ atoms. Insets show the bulk cohesive energy of $X$ or $Y$ bulk crystals. 

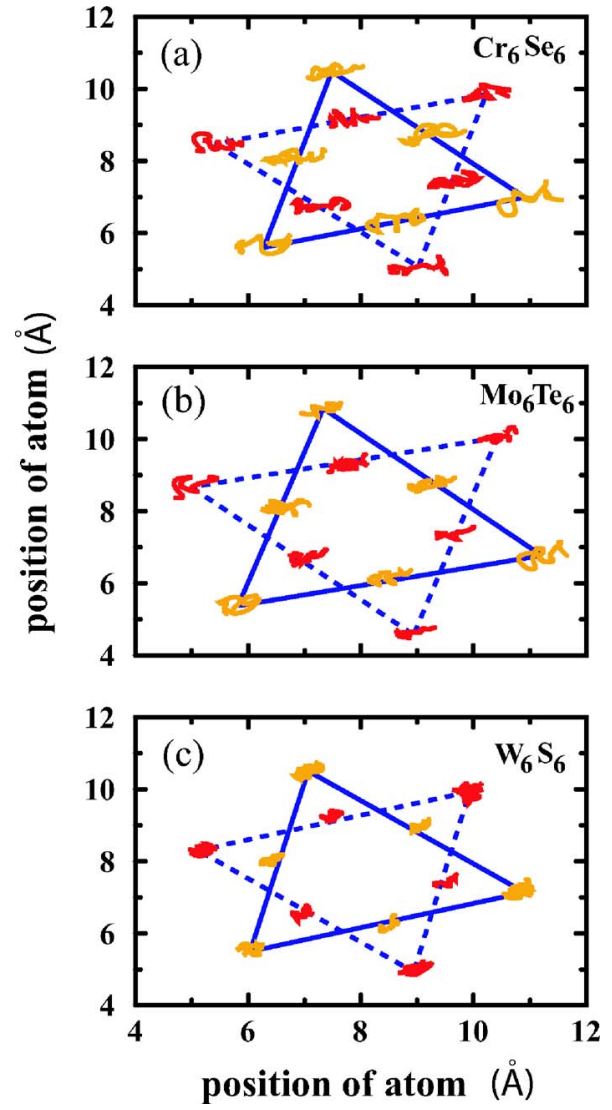

FIG. 3. (Color online) Trajectories of atoms around equilibrium position for one unit cell in (a) $\mathrm{Cr}_{6} \mathrm{Se}_{6}$, (b) $\mathrm{Mo}_{6} \mathrm{Te}_{6}$, and (c) $\mathrm{W}_{6} \mathrm{~S}_{6}$. Atoms in upper plane (lower) are labeled by orange (red) color.

found to be larger than the corresponding sum of atomic radii. In addition to these arguments, the calculated binding energy of $\mathrm{Li}_{2} \mathrm{Mo}_{6} \mathrm{Se}_{6}$ crystal relative to the energy of $\mathrm{Li}_{2} \mathrm{Mo}_{6} \mathrm{Se}_{6}$ nanowires is only $0.9 \mathrm{eV}$. Based on these results, we conclude that nanowires in the bulk crystal are held together mainly by moderate chemical interaction.

As a next step, we have carried out finite temperature $a b$ initio MD calculations for one type of nanowires in each metal group, namely $\mathrm{Cr}_{6} \mathrm{Se}_{6}, \mathrm{Mo}_{6} \mathrm{Te}_{6}$, and $\mathrm{W}_{6} \mathrm{~S}_{6}$. We have performed calculations in double cells at $500 \mathrm{~K}$ for 250 time steps (0.5 ps) using a Langevin thermostat. We also carried out $a b$ initio MD calculations in six unit cells (as a periodically repeating cell) at $500 \mathrm{~K}$ for 1 ps using a microcanonical ensemble but by scaling velocities at each time step. The Brillouin zone has been represented by a single $k$ point at $\Gamma$. These $a b$ initio MD calculations indicate that these wires are stable at high temperature. Trajectories of each atom in the course of MD simulations are illustrated around their equilibrium positions in Fig. 3. Maximum displacements of atoms from their equilibrium positions are highest (lowest) in $\mathrm{Cr}_{6} \mathrm{Se}_{6}\left(\mathrm{~W}_{6} \mathrm{~S}_{6}\right)$. Due to constraints of atomic motion at the corner of the inner triangle, deviation of chalcogen atoms from equilibrim are higher than metal atoms.

\section{ELECTRONIC PROPERTIES}

The detailed form of band structure around the Fermi energy $\left(E_{F}\right)$ determines the electronic properties of these mate-

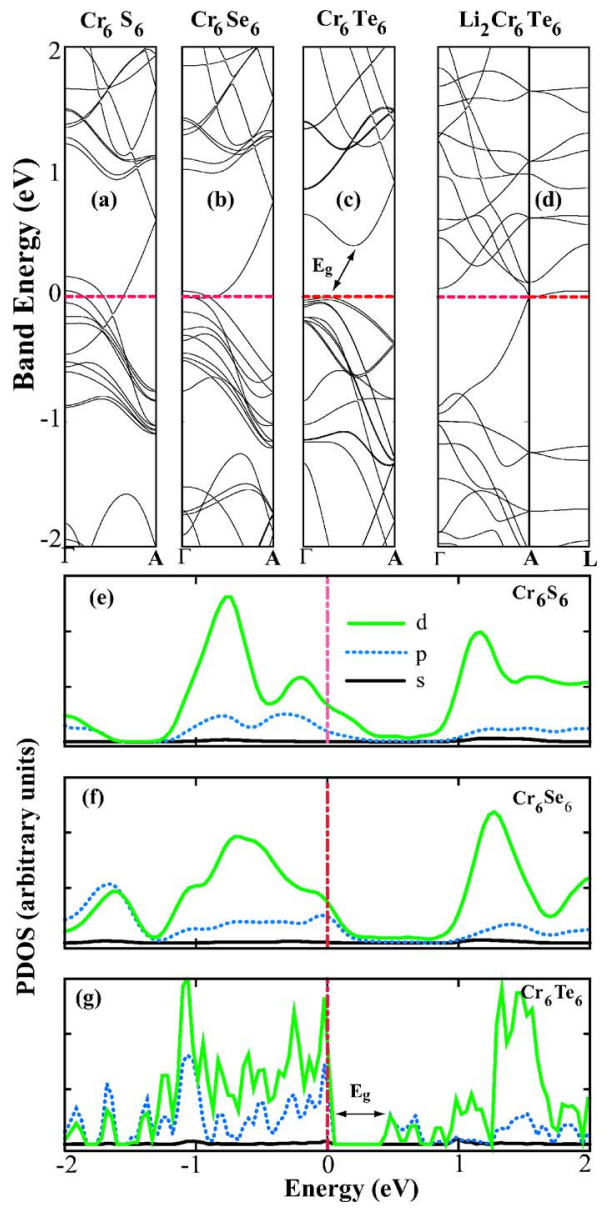

FIG. 4. (Color online) The band structure and partial density of states (PDOS) of $\mathrm{Cr}_{6} \mathrm{~S}_{6}$ (a), (e); $\mathrm{Cr}_{6} \mathrm{Se}_{6}$ (b), (f); $\mathrm{Cr}_{6} \mathrm{Te}_{6}$ (c), (g); and band structure of bulk $\mathrm{Li}_{2} \mathrm{Cr}_{6} \mathrm{Te}_{6}(\mathrm{~d})$. Fermi level of metallic systems shown by dashed lines marks the zero of energy. In semiconducting $\mathrm{Cr}_{6} \mathrm{Te}_{6}$, the zero of the energy indicates the top of the valence band. To resolve the band gap in $(\mathrm{g})$ the smearing of states is decreased. In contrast, the singularities of density of states disappeared owing to the smearing of states by the Gaussian function in (e) and (f).

rials. The number of bands which cross $E_{F}$ is crucial for the quantum ballistic conductance and the stability of nanowire. Under ideal conditions and within Landauer theory, the equilibrium conductance of an infinite wire would be determined by the number of bands $(n)$ crossing the $E_{F}$, namely $G$ $=2 n e^{2} / h$. However, in a recent study on $\mathrm{Mo}_{6} \mathrm{Se}_{6}$ nanowires, Luttinger liquid behavior has been observed ${ }^{16}$ experimentally. This suggests that conductance of $\mathrm{Mo}_{6} \mathrm{Se}_{6}$ nanowires may not be written in a simple Landauer form. Due to electron-electron interaction in 1D geometry, equilibrium conductance of defect-free infinite $\mathrm{Mo}_{6} \mathrm{Se}_{6}$ wire can be written as $\mathrm{G}=2 n K e^{2} / h$, where $K$ represents the dimensionless electron-electron interaction parameter of Luttinger liquid.

Our analysis indicates that the form of the energy band structure that belongs to each metal group is similar. In Fig. 4, only the band structure of isolated wires with $Y=\mathrm{Cr}$ are illustrated as prototypes. For $X=\mathrm{S}$ and Se cases, the isolated wires are metallic with three bands crossing $E_{F}$. Our partial density of states (PDOS) analysis presented in Fig. 4(e)-4(g) 
reveals that the bands crossing the $E_{F}$ have $d$-orbital character of $\mathrm{Cr}$ and $p$ character of $\mathrm{S}$ or $\mathrm{Se}$ atoms. However, the metallic $\mathrm{Cr}_{6} \mathrm{~S}_{6}$ and $\mathrm{Cr}_{6} \mathrm{Se}_{6}$ wires become semiconductors when chalcogen $\mathrm{S}$ or $\mathrm{Se}$ is replaced by Te to form $\mathrm{Cr}_{6} \mathrm{Te}_{6}$. In fact, all wires, $\mathrm{Cr}_{6} \mathrm{Te}_{6}, \mathrm{Mo}_{6} \mathrm{Te}_{6}, \mathrm{~W}_{6} \mathrm{Te}_{6}$, are found to be semiconductors. This behavior is attributed to the charge transfer between TM and chalcogen atoms. The Mulliken analysis shows that charge is normally transferred from TM atoms to the more electronegative $\mathrm{S}$ or Se atom. In the case of the Te atom, the direction of charge transfer is reversed. For example, while the $\mathrm{Cr}$ atom gives $0.17 e$ and $0.05 e$ to $\mathrm{S}$ and $\mathrm{Se}$, respectively, it takes $0.28 e$ from the Te atom. The trend is similar for other TM atoms (Mo, W). Due to charge transfer to the $\mathrm{Cr}$ atom from $\mathrm{Te}$, the electronic potential at $\mathrm{Cr}$ atom rises in energy. The energy gaps are $0.39,0.23$, and $0.25 \mathrm{eV}$ for $\mathrm{Cr}_{6} \mathrm{Te}_{6}, \mathrm{Mo}_{6} \mathrm{Te}_{6}$, and $\mathrm{W}_{6} \mathrm{Te}_{6}$, respectively. Figure 4(d) shows the band structure of bulk $\mathrm{Li}_{2} \mathrm{Cr}_{6} \mathrm{Te}_{6}$ along the $\Gamma$-A (along the wire) and A-L directions (perpendicular to the wire). In bulk systems, conduction electrons are localized on wires. The dispersion of bands along the $\Gamma$-A direction is stronger than the dispersion of bands along the A-L direction. This situation is consistent with the 1D nature of bulk $M_{2} Y_{6} X_{6}$ compounds. In this respect, our findings confirm the experimental results and other theoretical works. Very small band dispersions along a direction perpendicular to the wire indicate that the interaction between the individual chains is very weak. In the bulk case, alkali atoms $(\mathrm{Li}, \mathrm{Na})$ donate their electrons to wires and change the number of bands crossing the $E_{F}$. This charge transfer raises the $E_{F}$ and reduces the number of conduction bands of wires. For this reason, unlike $\mathrm{Cr}_{6} \mathrm{Te}_{6}$ nanowire, bulk $\mathrm{Li}_{2} \mathrm{Cr}_{6} \mathrm{Te}_{6}$ has metallic behavior.

\section{MECHANICAL PROPERTIES}

Our results concerning the stability and mechanical properties of the nanowires provide valuable information for the synthesis of these materials in the future. First, we present our extensive analysis of mechanical stability.

\section{A. Rotation from staggered to eclipsed geometry}

First, we have analyzed the stability of wires with respect to the rotation of the staggered triangular planes formed by $Y_{3} X_{3}$. In this study, one of the planes in the unit cell is fixed and the adjacent one is rotated in $12^{\circ}$ steps. At each step, only the lattice constant $c_{s c}=c$ is optimized. In Fig. 5, we

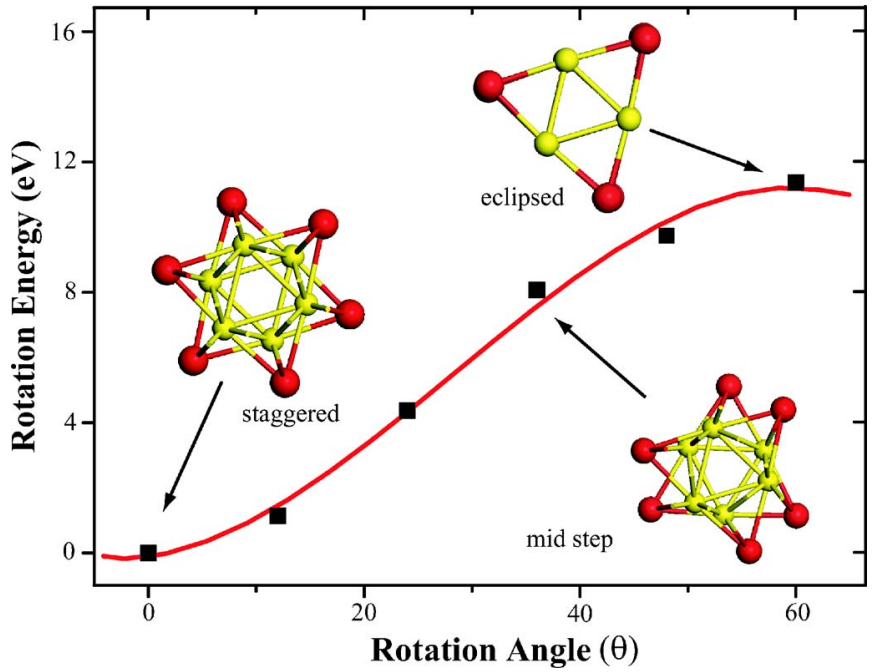

FIG. 5. (Color online) Variation of the total energy $E_{T}(\theta)$ of $\mathrm{Mo}_{6} \mathrm{Se}_{6}$ wire with the rotation angle $\theta$ between the adjacent triangles. The continuing line is fit to calculated data. The equilibrium total energy $E_{T}(\theta=0)$ is set to zero.

show the variation of rotation energy, i.e. $E_{T}(\theta)-E_{T}(\theta=0)$ as a function of rotation angle. Here, $E_{T}(\theta)$ denotes the total energy of a wire at a given rotation angle $\theta$. Calculations indicate that there is a high energy barrier $\Delta Q\left[=E_{T}(\theta=60)\right.$ $\left.-E_{T}(\theta=0)\right]$ between eclipsed and staggered structures. All wires prefer the staggered geometry and they relax from the eclipsed to the staggered geometry without any barrier. Interestingly, all isolated wires are nonmagnetic for all $\theta$.

\section{B. Elastic stiffness}

The axial strength of these wires has been investigated by calculating the elastic stiffness constant $\kappa$, which is defined as the second derivative of the strain energy per unit cell with respect to the axial strain $\epsilon_{z z}$, namely $\kappa=d^{2} E_{T} / d \epsilon_{z z}^{2}$. While calculating $\kappa$, we apply a small deformation to the wire along the wire axis in order to stay in the harmonic region. Consequently, we can use the Hooke's law to find restoring force. The calculated $\kappa$ values are listed in Table II. Most of them are higher than that of steel and also comparable with the theoretically predicted value for the $(8,0)$ single wall $\mathrm{Si}$ nanotube $\left(23 \mathrm{eV} /\right.$ atom). ${ }^{37}$ However, they are smaller than that of the single wall carbon nanotube as well as the linear carbon chain which are calculated to be

TABLE II. First row: Elastic stiffness constants. Second row: Minimum strain $\varepsilon_{z z, B}$ reqiured to break the isolated $Y_{6} X_{6}$ wire. Third row: Breaking-strain energy $E_{B}$ (or strain energy required until $\varepsilon_{z z, B}$ ) per cell. Fourth row: Ratio of strain energy to $\varepsilon_{z z, B}\left(E_{B} / \varepsilon_{z z, B}\right)$.

\begin{tabular}{lccccccccc}
\hline \hline & $\mathrm{Cr}_{6} \mathrm{~S}_{6}$ & $\mathrm{Cr}_{6} \mathrm{Se}_{6}$ & $\mathrm{Cr}_{6} \mathrm{Te}_{6}$ & $\mathrm{Mo}_{6} \mathrm{~S}_{6}$ & $\mathrm{Mo}_{6} \mathrm{Se}_{6}$ & $\mathrm{Mo}_{6} \mathrm{Te}_{6}$ & $\mathrm{~W}_{6} \mathrm{~S}_{6}$ & $\mathrm{~W}_{6} \mathrm{Se}_{6}$ & $\mathrm{~W}_{6} \mathrm{Te}_{6}$ \\
\hline$\kappa(\mathrm{eV} /$ cell $)$ & 192 & 117 & 156 & 280 & 254 & 210 & 306 & 254 & 220 \\
$\varepsilon_{z z, B}(\%)$ & 9.3 & 8.2 & 10.8 & 6.9 & 8.2 & 9.1 & 6.5 & 8.0 & 10.0 \\
$E_{B}(\mathrm{eV}$ per cell $)$ & 1.15 & 1.02 & 1.50 & 1.17 & 1.29 & 1.44 & 1.29 & 1.49 & 1.73 \\
$E_{B} / \varepsilon_{z z, B}(\mathrm{eV})$ & 0.13 & 0.13 & 0.14 & 0.17 & 0.16 & 0.16 & 0.20 & 0.19 & 0.18 \\
\hline \hline
\end{tabular}




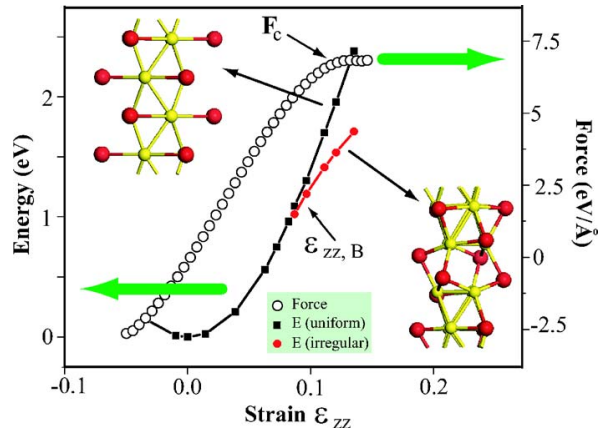

FIG. 6. (Color online) The variation of the total energy of $\mathrm{Cr}_{6} \mathrm{Se}_{6}$ with respect to the strain $\varepsilon_{z z}$ along the wire axis. Energy values indicated by square and filled circles correspond to the uniformly and irregularly elongating wire, respectively. The atomic structure of uniformly and irregularly elongating wires are given by left and right insets, respectively. The force variation of uniformly elongating wire is shown by empty circles. The maximum of force variation corresponds to the inflection point of the energy.

$52-60 \mathrm{eV} /$ atom $^{38}$ and $119 \mathrm{eV} /$ atom, ${ }^{39}$ respectively. In each metal group, the wire with a $\mathrm{S}$ atom is stiffer than others and the stiffness decreases from $\mathrm{S}$ to $\mathrm{Te}$ in the Mo and $\mathrm{W}$ group. This trend is not followed in the $\mathrm{Cr}$ case. In each chalcogen group, $\kappa$ tends to decrease from $\mathrm{W}$ to $\mathrm{Cr}$. These results indicate that $\kappa$ is related to the radii of atoms. Highest (lowest) value is obtained for largest (smallest) radius of a metal atom and smallest (largest) radius of a chalcogen atom.

\section{Breaking strain}

The variation of the total energy versus $\varepsilon_{z z}$ has been calculated for all wires by increasing $c_{0}$ uniformly in small steps. At each step, the structure has been fully relaxed. In Fig. 6, we illustrate our results for $\mathrm{Cr}_{6} \mathrm{Se}_{6}$. In the same figure, we also show the tensile force (minus the restoring force, i.e. $\left.F_{T}=\frac{1}{c_{0}} \partial E_{T} / \partial \varepsilon_{z z}\right)$. Here, one distinguishes two different behaviors. In the first one, the wires elongate uniformly under axial tensile force by conserving symmetry. Beyond a critical strain or maximum critical force $F_{c}$, a smaller force is required to achieve further elongation. This point coincides with the inflection point of the energy $E_{T}\left(\varepsilon_{z z}\right)$ curve in Fig. 6. This is taken usually as the breaking point of the atomic chains. Passing through the breaking point the wire expands uniformly but with a tensile force $F_{T}$ smaller than $F_{c}$.

In $Y_{6} X_{6}$ wires, we deduce also a behavior which is different from uniform elongation. Before one reaches the inflection point at a certain value of $\varepsilon_{z z}$, a structural transformation starts where bonds between TM atoms in different planes break, and concomitantly chalcogen atoms find a place between them. This structural transformation is derived by the elastic energy stored in the wire and is an anharmonic symmetry-breaking process. The system becomes more energetic by lowering the total energy $E_{T}$ whereby the wire expands irregularly. We notice that a similar anharmonic structural transformation after an elastic deformation took place in the stretching of metal nanowires with finite cross section. ${ }^{40}$ The transformation may release part of the elastic energy accumulated in the wire after a certain deformation. At the end, the cross section reduces at a local region and a neck develops. The onset of yielding is taken as the onset of breaking strain $\varepsilon_{z z, B}$ under constant tensile force. In Table II, we list calculated values of $\varepsilon_{z z, B}$, breaking-strain energy $E_{B}$, and $E_{B} / \varepsilon_{z z, B}$. Note that $E_{B}$ occurs in the range of $1-2 \mathrm{eV}$, which is typical for bond breaking energy.

Breaking strain $\varepsilon_{z z, B}$ of $\mathrm{Mo}_{6} \mathrm{~S}_{6}$ has been found to be $6.6 \%$ by Vilfan. ${ }^{18}$ Our calculated strain is $6.9 \%$ and in fair agreement with Vilfan. ${ }^{18}$ The breaking strain of $19 \%$ for $\mathrm{Mo}_{6} \mathrm{Se}_{6}$ has been found by Riberio et al. ${ }^{17}$ This value is about 2.5 times larger than our calculated breaking strain. In their calculations, a single cell has been used and the wire has expanded uniformly. We believe that this constraint gives rise to a larger breaking strain in Ref. 17. The value of the breaking strain $\varepsilon_{z z, B}$ increases with the row number of chalcogen atom in $\mathrm{Mo}_{6} X_{6}$ and $\mathrm{W}_{6} X_{6}$ wires.

\section{FUNCTIONALIZATION OF NANOWIRES}

We have next examined how the electronic and magnetic properties of $\mathrm{Mo}_{6} \mathrm{Se}_{6}$-type nanowires can be modified upon adsorption of foreign atoms. Adsorbate induced changes in the properties of nanowires are crucial for fabrication of chemical and biological sensors. Moreover, functional nanowires can be the building blocks of different devices. We choose $\mathrm{Mo}_{6} \mathrm{Se}_{6}$ which was synthesized experimentally as prototype and study the effects of $\mathrm{H}, \mathrm{O}, \mathrm{Cr}$ and $\mathrm{Ti}$ adsorption.

TABLE III. Calculated binding energies $E_{b}$ (spin polarized for $\mathrm{Cr}$ and $\mathrm{Ti}$ and spin unpolarized for $\mathrm{H}$ and $\mathrm{O}$ systems) in eV and net magnetic moment $\mu$ in $\mu_{B}$ Bohr magneton per adsorbate atom of individual atoms adsorbed on a possible four adsorption sites in a single cell and the most favorable binding site for a double cell of $\mathrm{Mo}_{6} \mathrm{Se}_{6}$ nanowire. Values in parentheses are the binding energies calculated relative to bulk or molecular energies of adatoms.

\begin{tabular}{|c|c|c|c|c|c|c|c|c|c|c|}
\hline \multirow[b]{3}{*}{ Atom } & \multicolumn{8}{|c|}{ Single cell } & & \\
\hline & \multicolumn{2}{|c|}{ site 1} & \multicolumn{2}{|c|}{ site 2} & \multicolumn{2}{|c|}{ site 3} & \multicolumn{2}{|c|}{ site 4} & \multicolumn{2}{|c|}{ Double cell } \\
\hline & $\mathrm{E}_{b}$ & $\mu$ & $\mathrm{E}_{b}$ & $\mu$ & $\mathrm{E}_{b}$ & $\mu$ & $\mathrm{E}_{b}$ & $\mu$ & $\mathrm{E}_{b}$ & $\mu$ \\
\hline $\mathrm{Cr}$ & 1.59 & 4.58 & 1.32 & 4.86 & 0.48 & 5.04 & 1.64 & 4.52 & $2.43(1.14)$ & 4.07 \\
\hline $\mathrm{Ti}$ & 2.94 & 2.18 & $2 \rightarrow 4$ & & 1.14 & 2.75 & 3.36 & 2.26 & $4.91(0.23)$ & 0.97 \\
\hline $\mathrm{O}$ & 3.76 & & 3.49 & & 2.27 & & $4 \rightarrow 1$ & & $3.82(-1.14)$ & \\
\hline $\mathrm{H}$ & 2.81 & & 2.88 & & 2.48 & & 2.96 & & $3.05(0.34)$ & \\
\hline
\end{tabular}




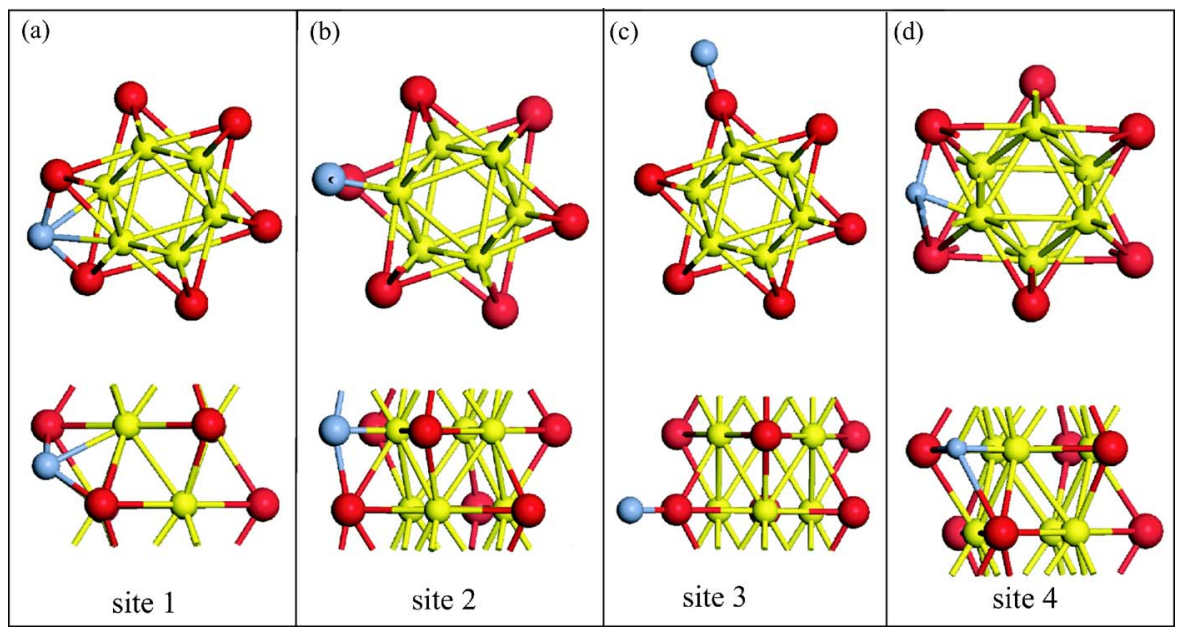

FIG. 7. (Color online) Possible adsorption sides on single cell $\mathrm{Mo}_{6} \mathrm{Se}_{6}$ wire. In each panel, first and second figures represent the top view and side view, respectively. The adatom (blue) is between $\mathrm{Mo}_{3} \mathrm{Se}_{3}$ planes in (a); adatom is bound to Mo atom (yellow) in (b); adatom is bound to Se atom (red) in (c), and in the same plane as $\mathrm{Mo}_{3} \mathrm{Se}_{3}$ unit in (d).
We have considered four different possible adsorption sites in a single cell and one site (which is the most favorable case) in a double cell of $\mathrm{Mo}_{6} \mathrm{Se}_{6}$ (see Fig. 7). In single cell calculations, the nearest adsorbate-adsorbate distance is equal to $\mathrm{c}_{0}(4.44 \AA)$, while this distance is $2 \mathrm{c}_{0}$ in double cell calculations. Therefore, single cell calculation allows a significant coupling between adjacent adatoms. This coupling is small in double cell configuration. $E_{b}$ of adsorbate atom in single cell configuration is obtained from the following expression:

$$
E_{b}=E_{T}\left[\mathrm{Mo}_{6} \mathrm{Se}_{6}\right]+E[A]-E_{T}\left[\mathrm{Mo}_{6} \mathrm{Se}_{6}+A\right],
$$

where $E_{T}\left[\mathrm{Mo}_{6} \mathrm{Se}_{6}\right]$ stands for the fully optimized total energy of bare $\mathrm{Mo}_{6} \mathrm{Se}_{6}$ nanowire calculated in single cell, $E[A]$ is the energy of the chain of adsorbate atom $\mathrm{A}$ and $E_{7}\left[\mathrm{Mo}_{6} \mathrm{Se}_{6}+A\right]$ is the fully relaxed total energy of the single adsorbate on $\mathrm{Mo}_{6} \mathrm{Se}_{6}$ nanowire. We note that $E[A]$ is calculated after removing the $\mathrm{Mo}_{6} \mathrm{Se}_{6}$ wire from the unit cell, but by keeping $\mathrm{A}$ in the same position as in $\mathrm{Mo}_{6} \mathrm{Se}_{6}+\mathrm{A} .{ }^{41}$ The binding energies $E_{b}$ are obtained according to the lowest ground-state total energies (either magnetic or nonmagnetic) of both $E_{T}\left[\mathrm{Mo}_{6} \mathrm{Se}_{6}+\mathrm{A}\right]$ and $E[A]$. Bare nanowire has a nonmagnetic ground state with zero net spin. All adsorbates studied here have positive $E_{b}$ which corresponds to optimized stable structure with significant binding. Our results are summarized in Table III. Both single and double cell calculations give the same site as the energetically most favorable site. The double cell calculations can be representative for single adsorption. While the A-A coupling energy is normally excluded in the $E_{b}$, the indirect effect of the A-A coupling to the chemisorption energy has remained. The bond between $\mathrm{A}$ and $\mathrm{Mo}_{6} \mathrm{Se}_{6}$ is generally weakened due to the charge transfer from the bond to the A-A atomic chain of adsorbates. In the present case, $E_{b}$ for $\mathrm{Ti}$ and $\mathrm{Cr}$ is 1.64 and $3.36 \mathrm{eV}$ per atom, respectively. These values are smaller than those obtained for double cell calculations. Both wires have magnetic ground states with $\mu=4.52$ and $2.26 \mu_{B}$ for $\mathrm{Cr}$ and $\mathrm{Ti}$, respectively. In $\mathrm{Ti}$ adsorption, initial geometry shown in Fig. 7(b) transforms to site 4 geometry shown in Fig. 7(d) after structural optimization is performed. In the $\mathrm{H}$ and $\mathrm{O}$ cases, $E_{b}$ is 2.96 and $3.76 \mathrm{eV}$, respectively. Initial site 4 geometry changes to site 1 in adsorption of $\mathrm{O}$. Binding of all adsorbates to site 3 is weaker than the other adsorption sites. It is also instructive to calculate the binding energies of adsorbates with respect to the total energies of bulk or molecular phases of these atoms instead of free atom energies. In Eq. (3), we have taken $E[\mathrm{~A}]$ as the total energy per atom for $\mathrm{Cr}$ and $\mathrm{Ti}$ crystals and molecular energy per atom for $\mathrm{H}$ and O. The resulting $E_{b}$ is represented in Table III in parentheses for double cell configuration. Even for this definition, significant $E_{b}$ is obtained except for O. For oxygen, the reaction is endothermic. In double cell calculations, the amount of change in net magnetic and $E_{b}\left(\Delta E_{b}\right)$ with respect to single cell calculations are different for each adatom. Dramatic change of induced net magnetic moment and $E_{b}$ is observed in Ti adsorption. $\Delta E_{b}$ is $1.55 \mathrm{eV}$ for $\mathrm{Ti}$ and $0.84 \mathrm{eV}$ for $\mathrm{Cr}$. Initial structure of wire is not preserved upon adsorption. Bond distances at the proximity of the adatom are slightly disturbed. For example, the nearest Mo-Se in-plane bond length extends from 2.61 to $2.74 \AA$ in the case of Cr absorption and from 2.61 to $2.92 \AA$ for the Ti case. Metallic behavior does not change by these adsorbates.

\section{CONCLUSION}

We have found that not only $\mathrm{Mo}_{6} \mathrm{Se}_{6}$, but also transition metal atoms in the same column as Mo and chalcogen atoms in the same column as Se can form $\mathrm{Mo}_{6} \mathrm{Se}_{6}$-type stable nanowires. All wires have a high binding energy and it increases with increasing (decreasing) row number of metal (chalcogen) atoms. The molecular dynamics simulations performed at $500 \mathrm{~K}$ and relaxation after random displacement of atoms indicate the stability of these structures. The wires with Te as a chalcogen atom are semiconductors but the rest of the family is metallic. Band structure for the bulk case gives rise to strong dispersion along wire axis. The calculated elastic stiffness constants point out that these wires are strong. Adatoms such as, $\mathrm{O}, \mathrm{H}, \mathrm{Cr}$, and $\mathrm{Ti}$ atoms are also strongly bound to the $\mathrm{Mo}_{6} \mathrm{Se}_{6}$ wire. Among them, $\mathrm{Cr}$ and $\mathrm{Ti}$ induce magnetic moment. 
*Electronic address: ciraci@fen.bilkent.edu.tr

${ }^{1}$ S. Ciraci, A. Buldum, and I. P. Batra, J. Phys.: Condens. Matter 13, R537 (2001).

${ }^{2}$ H. Ohnishi, Y. Kondo, and K. Takayanagi, Nature (London) 395, 780 (1998).

${ }^{3}$ O. Gülseren, F. Ercolessi, and E. Tosatti, Phys. Rev. Lett. 80, 3775 (1998).

${ }^{4}$ S. Iijima, Nature (London) 354, 56 (1991); S. Iijima, T. Ichihashi, and Y. Ando, ibid. 356, 776 (1992).

${ }^{5}$ M. S. Dresselhaus, G. Dresselhaus, and P. C. Eklund, Science of Fullerenes and Carbon Nanotubes (Academic, San Diego, CA, 1996).

${ }^{6}$ S. Ciraci, S. Dag, T. Yildirim, O. Gülseren, and R. T. Senger, J. Phys.: Condens. Matter 16, R1 (2004).

${ }^{7}$ R. T. Senger, S. Dag, and S. Ciraci, Phys. Rev. Lett. 93, 196807 (2004).

${ }^{8}$ S. Dag and S. Ciraci, Phys. Rev. B 71, 165414 (2005).

${ }^{9}$ S. Dag, E. Durgun, and S. Ciraci, Phys. Rev. B 69, 121407(R) (2004).

${ }^{10}$ Y. Zhang and H. Dai, Appl. Phys. Lett. 77, 3015 (2000); Y. Zhang, N. W. Franklin, R. J. Chen, and H. Dai, Chem. Phys. Lett. 331, 35 (2000).

${ }^{11}$ M. Potel, R. Chevrel, M. Sergent, J. C. Armici, M. Decroux, and O. Fischer, J. Solid State Chem. 35, 286 (1980).

${ }^{12}$ J. M. Tarascon, F. J. DiSalvo, and J. V. Warszczak, Solid State Commun. 52, 227 (1984); and J. M. Tarascon, G. W. Hull, and F. J. DiSalvo, Mater. Res. Bull. 19, 915 (1984).

${ }^{13}$ J. H. Golden, F. J. DiSalvo, J. M. Fréchet, J. Silcox, M. Thomas, and J. Elman, Science 273, 782 (1996).

${ }^{14}$ B. G. M. Chew, J. H. Golden, B. A. Huggins, F. J. DiSalvo, and D. B. Zax, Phys. Rev. B 50, 7966 (1994).

${ }^{15}$ L. Venkataraman and C. M. Lieber, Phys. Rev. Lett. 83, 5334 (1999).

${ }^{16}$ L. Venkataraman, Y. S. Hong, and P. Kim, Phys. Rev. Lett. 96, 076601 (2006).

${ }^{17}$ F. J. Riberio, D. J. Roundy, and M. L. Cohen, Phys. Rev. B 65, 153401 (2002).

${ }^{18}$ I. Vilfan, Eur. Phys. J. B 51, 277 (2006).

${ }^{19}$ S. Gemming, G. Seifert, and I. Vilfan, Phys. Status Solidi B 243, 3320 (2006).

${ }^{20}$ M. F. Yu, B. S. Files, S. Arepalli, and R. S. Ruoff, Phys. Rev. Lett. 84, 5552 (2000).
${ }^{21}$ S. Ogata and Y. Shibutani, Phys. Rev. B 68, 165409 (2003).

${ }^{22}$ A. Meden, A. Kodre, J. P. Gomilsek, I. Arcon, I. Vilfan, D. Vrbanic, A. Mrzel, and D. Mihailovic, Nanotechnology 16, 1578 (2005).

${ }^{23}$ T. Yang, S. Okano, S. Berber, and D. Tománek, Phys. Rev. Lett. 96, 125502 (2006).

${ }^{24}$ Xiubin Qi and Frank E. Osterloh, J. Am. Chem. Soc. 127, 7666 (2005).

${ }^{25}$ Xiubin Qi, Frank E. Osterloh, S. A. Barriga, J. A. Giacomo, and S. Chiang, Anal. Chem. 127(4), 1306 (2006).

${ }^{26}$ M. C. Payne, M. P. Teter, D. C. Allen, T. A. Arias, and J. D. Joannopoulos, Rev. Mod. Phys. 64, 1045 (1992).

${ }^{27}$ Numerical computations have been carried out by using VASP software: G. Kresse and J. Hafner, Phys. Rev. B 47, R558 (1993); G. Kresse and J. Furthmuller, ibid. 54, 11169 (1996).

${ }^{28}$ P. Hohenberg and W. Kohn, Phys. Rev. 136, B864 (1964); W. Kohn and L. J. Sham, Phys. Rev. 140, A1133 (1965).

${ }^{29}$ D. Vanderbilt, Phys. Rev. B 41, R7892 (1990).

${ }^{30}$ J. P. Perdew, J. A. Chevary, S. H. Vosko, K. A. Jackson, M. R. Pederson, D. J. Singh, and C. Fiolhais, Phys. Rev. B 46, 6671 (1992).

${ }^{31}$ M. Methfessel and A. T. Paxton, Phys. Rev. B 40, 3616 (1989).

${ }^{32}$ H. J. Monkhorst and J. D. Pack, Phys. Rev. B 13, 5188 (1976).

${ }^{33}$ The crystal structure of $\mathrm{Se}$ and $\mathrm{Te}$ have hexagonal lattices in which the atoms are arranged in spiral chains oriented along the $z$ axis. S has face centered orthorhombic crystal structure.

${ }^{34}$ Calculated bulk cohesive energies and lattice constants are in agreement with experimantel values.

${ }^{35}$ C. Kittel, Introduction to Solid State Physics, 7th ed. (Wiley, New York, 1996).

${ }^{36}$ D. M. Ceperley and B. J. Alder, Phys. Rev. Lett. 45, 566 (1980).

${ }^{37}$ E. Durgun, S. Tongay, and S. Ciraci, Phys. Rev. B 72, 075420 (2005).

${ }^{38}$ D. Sánchez-Portal, Emilio Artacho, José M. Soler, A. Rubio, and P. Ordejon, Phys. Rev. B 59, 12678 (1999).

${ }^{39}$ S. Tongay, R. T. Senger, S. Dag, and S. Ciraci, Phys. Rev. Lett. 93, 136404 (2004).

${ }^{40}$ H. Mehrez and S. Ciraci, Phys. Rev. B 56, 12632 (1997).

${ }^{41}$ Since $E[A]$ is not a single free atom energy, but the energy of an atomic chain having lattice parameter of the cell, it includes the A-A coupling. Therefore A-A coupling is excluded from $E_{b}$. 